\title{
COMPARACIÓN DE METODOLOGÍAS EN APLICACIONES WEB
}

\section{COMPARISON OF METHODOLOGIES IN WEB APPLICATIONS}

\author{
Jimmy Rolando Molina Ríos ${ }^{1}$ \\ Mariuxi Paola Zea Ordóñez ${ }^{2}$ \\ María José Contento Segarra ${ }^{3}$ \\ Fabricio Gustavo García Zerda ${ }^{4}$
}

1. Universidad Técnica de Machala. Grupo de Investigación de la carrera de Ingeniería de Sistemas (Ecuador).E-mail: jmolina@utmachala.edu.ec

2. Universidad Técnica de Machala. Grupo de Investigación de la carrera de Ingeniería de Sistemas (Ecuador).E-mail: mzea@utmachala.edu.ec

3. Universidad Técnica de Machala. Grupo de Investigación de la carrera de Ingeniería de Sistemas (Ecuador).E-mail: mjcontento est@utmachala.edu.ec

4. Universidad Técnica de Machala. Grupo de Investigación de la carrera de Ingeniería de Sistemas (Ecuador). E-mail: fggarciaz est@utmachala.edu.ec

\section{Citación sugerida:}

Molina Ríos, J.R., Zea Ordóñez, M.P., Contento Segarra, M.J. y García Zerda, F.G. (2018). Comparación de metodologías en aplicaciones web. 3C Tecnología: glosas de innovación aplicadas a la pyme, 7(1). 1-19. DOI: <http://dx.doi.org/10.17993/3ctecno.2018.v7n1e25.1-19/>. 


\section{RESUMEN}

En la actualidad, las aplicaciones Web están acaparando el mercado de los productos de software debido a las múltiples ventajas que ofrecen y a la dependencia del internet para el desarrollo de todo tipo de operación, dejando de lado a las típicas aplicaciones de escritorio. Esto se debe a la estructura cerrada que presentan y a la poca flexibilidad ante los cambios constantes de la tecnología. No obstante, ante el crecimiento de este mercado, los problemas referentes al desarrollo de estas aplicaciones se hacen cada vez más frecuentes, razón por la cual han surgido numerosas metodologías que permiten guiar el ciclo de vida del software y que además ayudan a mitigar y resolver los errores existentes respecto a cada etapa de desarrollo. Por tal motivo, se realizó una investigación exhaustiva para obtener información sobre las metodologías y cuáles de éstas según diversos autores provee características esenciales en el desarrollo Web, por lo que se plantea el objetivo de comparar las metodologías de desarrollo de software Web a través de la recolección de información bibliográfica, analítica y de campo. Como resultado de la investigación se ha podido identificar que la metodología OOHDM, es la que provee las mejores características para el campo del desarrollo de aplicaciones Web, siendo además la más utilizada entre programadores y empresas desarrolladores de software en la provincia del ORO.

\section{ABSTRACT}

Currently, Web applications are monopolizing the market of software products due to the multiple advantages they offer and the dependence on the internet for the development of all types of operations, leaving aside the typical desktop applications. This is due to the closed structure that already have little flexibility in the face of constant changes in technology. However, in view of the growth of this market, the problems related to the development of these applications are becoming more frequent, which is why numerous methodologies have emerged to guide the life cycle of software and also help to mitigate and resolve the existing errors regarding each stage of development. For this reason, an exhaustive investigation was carried out to obtain information on the methodologies and which of these according to diverse authors provides essential characteristics in the Web development, for which the objective is to compare the methodologies of Web software development through the Collection of bibliographic, analytical and field information. As a result of the research it has been possible to identify that the OOHDM methodology is the one that provides the best features for the field of Web application development, being also the most used among programmers and software developers in the province of ORO.

\section{PALABRAS CLAVE}

Comparación, Análisis, Metodologías web, Aplicaciones web, OOHDM.

\section{KEY WORDS}

Comparison, Analysis, Web Methodologies, Web Applications, OOHDM. 


\section{INTRODUCCIÓN}

Las metodologías de desarrollo de software surgen como un alternativa y marco de trabajo a partir de la complejidad que conlleva realizar un software y como respuesta ante los problemas que se presentaban en cada etapa de desarrollo debido a que no se lleva un control adecuado lo que generaba problemas y desarrollo de software deficientes que no cumplían los requisitos del cliente.

En la actualidad, el desarrollo de aplicaciones Web ha surgido exponencialmente debido al impacto del internet en el mundo como medio de difusión de información y demás servicios. La complejidad de desarrollo de las aplicaciones Web se ha incrementado con los avances tecnológicos en el campo de la programación, así también han surgido diversos problemas como los procesos de manejo de grandes volúmenes de información, cambios en las especificaciones del software, falta de comunicación, y deficiencia en la seguridad, por lo que es indispensable que cada empresa que trabaje en el campo de desarrollo de trabaje una metodología.

Con la implementación de las metodologías dentro del ciclo de vida de las aplicaciones Web se ha logrado no solo mejorar el proceso de creación y desarrollo de software, sino también se ha disminuido el nivel de riesgo, puesto que cada metodología ofrece una guía compuesta por etapas y procesos efectivos que permiten obtener resultados de calidad.

Considerando lo mencionado, en esta investigación de tipo bibliográfica, analítica y de campo se presenta una comparación de las metodologías Web basada en criterios de calidad y criterios específicos los cuales abarcan aspectos como representación gráfica, niveles de diseño, ciclo de vida, tipos de notación, estándares, tipo de modelado y herramientas de soporte. Las metodologías que se utilizaron para la comparación son: SOHDM, OOHDM, WSDM, WAE, UWE y IWEB.

En base a esta comparativa se puede verificar que las metodologías más utilizadas abarcan una mayor cantidad de criterios y/o elementos de casi todas las metodologías. Estas permiten concentrar sus esfuerzos en aspectos Web a diferencia de las otras las cuales se centran en brindar soluciones a problemas de carácter específico.

A partir del análisis de la información y comparación en el marco de desarrollo del artículo se obtuvo como resultados que la metodología OOHDM es la que cumple con casi todas las características para el proceso de desarrollo de aplicaciones Web. Además de que esta también se consideró dentro de los programadores y desarrolladores como la más óptima para guiar todo el proceso. 


\section{FUNDAMENTOS TEÓRICOS}

\subsection{DESARROLLO DE SOFTWARE}

Según Carmen de Pablos Heredero, el software es un "conjunto de elementos en interacción dinámica organizados para la consecución de un objetivo" [1] y en complemento con el hardware permiten la realización de diversas tareas de manera ágil. Por ello, el desarrollo de software ha aumentado notablemente en diferentes ámbitos de aplicación, es por ello que antes de crear una nueva aplicación debe tomarse en cuenta un sin números de aspectos, características y funciones que contendrá el nuevo producto [2] [3]. Según Pressman "se concluye que debe hacerse un esfuerzo concentrado para entender el problema antes de desarrollar una aplicación de software" [4].

\subsection{APLICACIONES WEB}

"Las aplicaciones Web no son más que herramientas de ofimática de la Web 2.0 que se manejan simplemente con una conexión a internet, y en estos casos cabe la opción de utilizar el ordenador solo como forma de procesos de la aplicación remota" [5].

"Una aplicación Web (Web based application) es una aplicación cliente/servidor, donde tanto el cliente (el navegador, explorador o visualizador) como el servidor (el servidor Web) y el protocolo mediante el que se comunican (HTTP) están estandarizados y no han de ser creados por el programador de aplicaciones" [6].

En la actualidad, las aplicaciones Web son cada vez más populares y su uso ha acaparado los ámbitos científico, cultural, académico, empresarial entre otros, y esto es debido a las múltiples ventajas que el usuario tiene respecto a los programas de escritorio. Entre otras, las ventajas que podemos mencionar son: sistema operativo multiplataforma, ejecutadas por cualquier dispositivo informático que tenga conexión a internet, no requiere de la instalación de programas solo un navegador, las copias de seguridad son almacenadas en los servidores, la información que se genera puede ser compartida de forma simultanea por varias personas, el espacio ocupado por los datos está a cargo del servidor y es de fácil uso [7].

\subsection{METODOLOGÍA PARA EL DESARROLLO DE SOFTWARE}

La construcción de software es una actividad que debe ser planificada y sistematizada mediante estrategias que garanticen el éxito del mismo. Es muy probable que surja cualquier inconveniente en el transcurso o al final del desarrollo tales como: requisitos cambiantes, planificaciones o presupuestos que no son realistas, falta de personal y clientes insatisfechos que conlleven al fracaso. [8] "El desarrollo de software no es una tarea fácil. Como resultado a este problema ha surgido una alternativa desde hace mucho: la Metodología" [9].

Una metodología de desarrollo de software es un proceso o conjunto de procedimientos, técnicas y documentación que permiten a los desarrolladores guiar y ejecutar el proyecto con el objetivo de crear nuevas aplicaciones de calidad que satisfagan las expectativas del 
DOI: http://dx.doi.org/10.17993/3ctecno.2018.v7n1e25.1-19

cliente. Según Pressman define a la metodología como "un marco de trabajo usado para estructurar, planificar y controlar el proceso de desarrollo de sistemas computaciones" [10].

\subsection{METODOLOGÍAS TRADICIONALES}

Las metodologías tradicionales aparecieron en la década de los 60, debido a un desarrollo de software totalmente manual con la necesidad de optimizar los procesos y objetivos propuestos en los proyectos de desarrollo, "se centran especialmente en el control del proceso, estableciendo rigurosamente las actividades involucradas, los artefactos que se deben producir, y las herramientas y notaciones que se usarán" [11].

Estas metodologías dividen el proceso o desarrollo del sistema en diversas etapas, lo cual permite la retribución de funciones a los miembros del equipo de desarrollo y, de esta manera, establecen el rehúso de componentes. También fomentan la documentación de las aplicaciones desarrolladas, por lo tanto garantiza una fácil comprensión y mantenimiento de las mismas.

\subsection{METODOLOGÍAS ÁGILES}

Según Andrés Navarro Cadavid [12], las metodologías ágiles son flexibles es decir pueden ser fácilmente modificadas en el caso que el equipo desarrollador o el proyecto lo requiera. Estas metodologías permiten subdividir el proyecto en pequeñas fracciones y mediante esto ser desarrollado de manera autónoma en un corto lapso de tiempo estimado entre dos a seis semanas. Son adaptables a los cambios de los requisitos por parte del cliente, entregan prototipos constantemente de tal manera que se garantiza un mejor producto. Fomenta el trabajo en equipo considerando al cliente parte del mismo.

\subsection{METODOLOGÍAS HÍBRIDAS}

Del sin número de metodologías que existen, ya sean estas agiles o tradicionales, surgen las metodologías híbridas, como una combinación de las dos anteriores, pero en este caso rescatando las prioridades que se destacan las metodologías mencionadas con el propósito de crear un método firme y flexible que se adapte a todo tipo de proyectos para el desarrollo de software.

Las metodologías híbridas "pretenden retomar las ventajas de las metodologías existentes, de tal forma que son una combinación de las mejores prácticas descritas en cada una de ellas." [14]. Este tipo de metodologías también pueden desarrollarse con la unión de cada una de las mejores características que existen dentro del mismo tipo de metodología.

\subsection{METOdOLOgÍAs PARA EL DESARROLLO DE APLICACIONES WEB}

Las metodologías o también llamados métodos, están compuestos por un número de fases que cambia dependiendo de las metodologías que se utilice, pero si el método es más 
complejo en sus pasos mayormente se tiene un menor número de errores en relación a la calidad y consistencia de datos.

Las metodologías para aplicaciones Web contienen fases para el desarrollo de software que pueden aumentar o disminuir dependiendo del método que utilicen, según Nieves del Valle [15] la mayoría de los métodos coinciden en las siguientes etapas:

- Diseño Conceptual: en esta sección se abarca temas relaciones a la especificación del dominio del problema, a través de su definición y las relaciones que contrae.

- Diseño Navegacional: está enfocado en lo que respecta al acceso y forma en la que los datos son visibles.

- Diseño de la presentación o diseño de interfaz: se centra en la forma en la que la información va a ser mostrada a los usuarios, cabe mencionar en esta sección intervienen mayormente el cliente definiendo los requerimientos y lo usuarios defiendo como quieren interactuar con el sistema.

- Implantación: es la construcción del software a partir de los artefactos generados en las etapas previas.

A continuación, se analizarán varias metodologías de desarrollo Web en conjunto con las etapas de cada uno de ellas.

\subsection{WSDM (WEB DESIGN METHOD)}

Es una metodología netamente para aplicaciones Web, hoy en día las aplicaciones deben desarrollarse en un lapso corto de tiempo siguiendo su estructura semántica del contenido y funcionalidad. Es por esto que se la considera apropiada para aplicaciones Web. Sin embargo, no es recomendada para la gestión de proyectos, para lo cual se debe utilizar una metodología adicional que facilite el ciclo de vida del software [16].

\subsection{FASES DE LA METODOLOGÍA WSDM}

Los autores [17], [18], [19], coinciden que la metodología WSDM, es una propuesta de desarrollo de aplicaciones Web que se enfoca en el usuario, y considera que este es el principal actor del sistema, es el encargado de definir lo requisitos, y los usuarios que interviene en la aplicación.

En WSDM se clasifica a los usuarios, y en base a ello se definen parámetros diseño. Esta metodología se enfoca más en sitios Web estáticos que brindan información que sitios o aplicaciones dinámicas. Asimismo, no ha tenido tanta acogida, debido que como se explicó anteriormente solo se centra en los usuarios y no tanto en los datos, otro aspecto importante en el desarrollo de aplicaciones Web.

WSD consta de tres capas: contexto (establecimiento de requerimientos), navegación (interacción sistema-usuarios), e información (datos de los usuarios de carácter relevante). En cuanto a las fases de modelado para la implementación de una aplicación, la WSDM establece 4 fases o actividades que se especifican en la ilustración 1. 


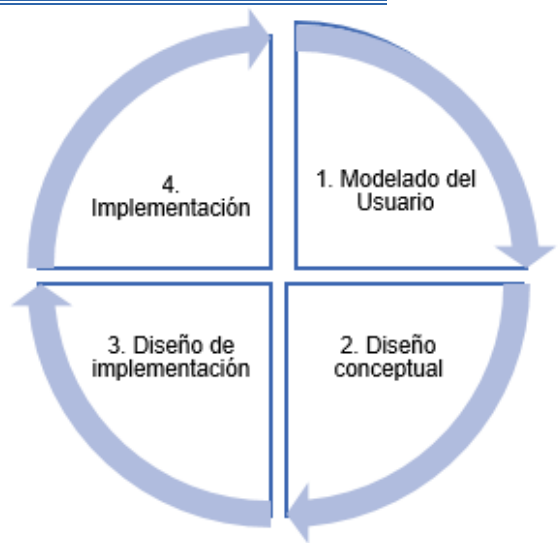

Ilustración 1. Fases de la metodología WSDM.

A continuación, se realizará una breve descripción de las fases de esta metodología.

- Modelado de Usuario: sirve para identificar a los posibles usuarios de la aplicación y la información que ellos requerirían de este sitio.

- Diseño conceptual: se desarrolla el modelado conceptual, organiza la información, se clasifica a los usuarios, se modela los objetos, se crea diagramas entidad-relación y crea el diseño navegacional. Cada diseño de navegación en el sitio Web será diferente por cada perfil usuario y por ende tendrá su propia perspectiva. Los entregables de esta fase son el modelo conceptual, y diseño navegacional.

- Diseño de implementación: se crea un diseño en base a los requerimientos del usuario, este prototipo de interfaz del sitio Web deberá tener una apariencia agradable, ser eficiente y seguro, así mismo aquí se especifican las restricciones de diseño, según lo que se estableció en el diseño conceptual.

- Implementación: se realiza la selección del entorno de desarrollo, construcción de la arquitectura, codificación y verificación de la funcionalidad total de la aplicación Web.

\subsection{SOHDM (SCENARIO-BASED OBJECT-ORIENTED HYPERMEDIA DESIGN METHODOLOGY)}

Es una metodología orientada a objetos en hipermedia que desarrolla diseños en escenarios o panoramas. Además, permite capturar las necesidades del sistema proponiendo el uso de escenario. SOHDM parte de un diagrama donde se identifican las entidades externas capaces de comunicarse con el sistema, es una metodología muy parecida a la metodología OOHDM diferenciadas por la utilización de escenarios. [20]

Escalona [21] declara que SOHDM propone el uso de escenarios por cada evento diferente, con el fin de conocer cuáles son las necesidades del sistema. Cada escenario simboliza el proceso de interacción que existe entre el usuario y el sistema, en este proceso se detallan los objetos involucrados, el flujo de actividades, y las operaciones realizadas. A partir de cada escenario se puede obtener el modelo conceptual, el mismo que se refleja en un diagrama de clases. 
En cuanto a los procesos de gestión de desarrollo de software o ciclo de vida Solís \& Vilariño [15], [16] describen 6 fases las cuales se pueden observar en la ilustración 2.

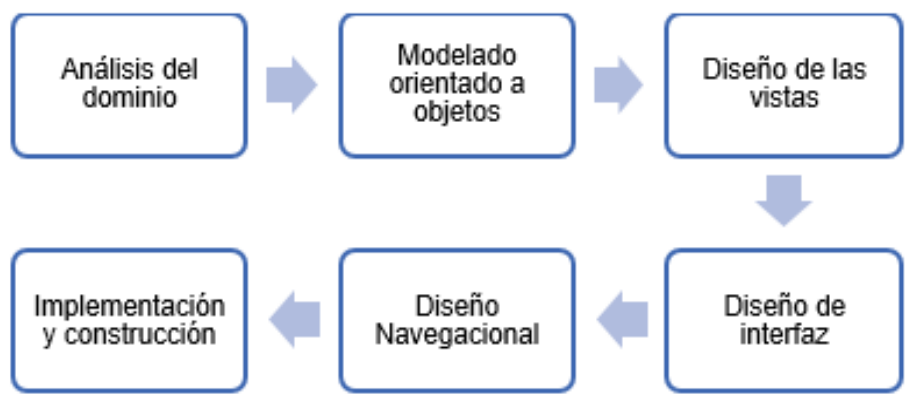

Ilustración 2. Fases de la metodología SOHDM.

- Análisis del dominio: establece los límites de la aplicación que se desarrollará, y se los representa mediante un diagrama de flujo. Además, se hace uso de los SACs (Scenarios activity charts) que no son más que escenarios donde se determina los requisitos de la aplicación.

- Modelo de objetos: en esta etapa se utilizan los SACs para realizar el modelado de objetos. Los usuarios son los principales objetos del sistema, cada usuario es descrito en el documento de desarrollo de la aplicación, los cuales incluyen atributos, asociaciones y cardinalidad.

- Diseño de las vistas: se representa las vistas por medio de unidades de navegación, cada vista agrupa información de las clases de la aplicación.

- Diseño navegacional: Identifica la navegación de los objetos dentro de la aplicación Web y la forma en cómo interactúan a través de enlaces, menús, nodos, consultas, entre otros.

- Implementación: se genera la interfaz de la aplicación, la lógica de negocio y el esquema de la base de datos.

- Construcción: desarrollo de la aplicación final, la cual cumple con todas las necesidades y requerimientos que fueron establecidos inicialmente por los usuarios.

\subsection{OOHDM (OBJECT ORIENTED HYPERMEDIA DESIGN METHODOLOGY)}

Según Soto, Palma \& Roncagliolo [22] OOHDM es una metodología orientada a objetos que propone un proceso de desarrollo de cinco fases donde se combinan notaciones gráficas UML con otras propias de la metodología.

Cuando internet no era accesible para todas las personas OOHDM simplemente era utilizado para aplicaciones hipermedia, pero gracias al auge del internet en la actualidad se adaptó dicha metodología para el desarrollo de aplicaciones hipermedias orientadas a la Web, como por ejemplo bibliotecas virtuales, sitios educativos, motores de búsqueda, entretenimiento, etc.

Esta metodología permite desarrollar aplicaciones Web a partir de la utilización de modelos especializados como: conceptual, navegación e interfaz de usuario teniendo como objetivo simplificar y hacer más eficaz el diseño de aplicaciones. 


\subsection{FASES DE LA METODOLOGÍA OOHDM}

A continuación, se describirán las cinco etapas de la metodología OOHDM.

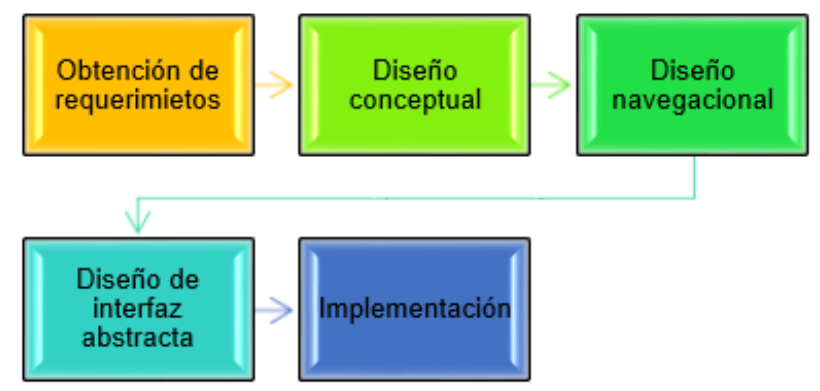

llustración 3. Fases de la metodología OOHDM.

- Obtención de requerimientos: se plantea la obtención de requerimientos de manera cuidadosa, entonces es muy importante conocer los actores y tareas que se deben modelar en los casos de uso.

- Diseño conceptual: se representa el modelo conceptual a través del modelamiento de diagramas de clases basados en clases, relaciones y subsistemas, enfocándose en el dominio semántico dejando de lado a los actores y tareas.

- Diseño navegacional: representa los diferentes caminos que puede ejecutar la aplicación dependiendo del tipo de usuario. Es decir, brinda un contexto navegacional capaz de realizar acciones a través de enlaces, vínculos o índices que están relacionados dentro de la aplicación Web dependiendo del perfil de usuario para mostrar sus vistas correspondientes.

- Diseño de interfaz abstracta: es ejecutada después del diseño navegacional, donde es necesario especificar las interfaces de usuario que se visualizaran en la aplicación Web. Dentro de este modelo se pueden identificar dos sub-tareas tales como el diseño estructural y el diseño de comportamiento.

- Implementación: implementar la aplicación Web independientemente de la plataforma que será utilizada. Esta fase también es conocida como puesta en marcha ya que es partir de aquí en donde los usuarios empiezan a utilizar y sacar provecho al sistema elaborado, a través de un navegador Web y conexión a internet.

Es importante mencionar que el desarrollador y diseñador son los encargados del lado técnico del sistema y su apariencia final, mientras que el cliente verifica que funcione correctamente como lo ha solicitado en la primera fase o etapa. 
DOI: http://dx.doi.org/10.17993/3ctecno.2018.v7n1e25.1-19

\begin{tabular}{|c|c|c|}
\hline ETAPAS & PRODUctos & FORMALISMOS \\
\hline Obtención de requerimientos & Casos de Uso (actores, escenarios) & $\begin{array}{c}\text { Plantillas del formato del documento, } \\
\text { Diagramas de Interacción de Usuario (UIDs). }\end{array}$ \\
\hline Diseño conceptual & Clases, subsistemas, relaciones, atributos & Modelos Orientados a Objetos. \\
\hline Diseño navegacional & Nodos, enlaces, estructuras de acceso, \\
contextos, navegacionales, & Vistas Orientadas a Objetos, Cartas de \\
transformaciones de navegación. & Contexto. \\
\hline Diseño de interfaz Abstracta & $\begin{array}{c}\text { Objetos de la interfaz abstracta respuestas } \\
\text { a eventos externos, transformaciones de la } \\
\text { interfaz. }\end{array}$ & $\begin{array}{c}\text { Vistas Abstractas de Datos (ADV), Diagramas } \\
\text { de Configuración Cartas de navegación de los } \\
\text { ADVs. }\end{array}$ \\
\hline Implementación & Aplicación en funcionamiento. & Los soportados por el entorno. \\
\hline
\end{tabular}

Tabla 1. Productos y formalismos de la metodología OOHDM.

\subsection{WAE (WEB APPLICATION EXTENSION)}

La WAE es una extensión de UML, que no se enfoca en el paradigma orientado a objetos si no en los elementos Web. WAE incorpora algunos conceptos como JavaScript y Form. En esta metodología cubre el lado tanto del servidor (páginas del servidor) como el cliente (Active $X$, applet Java, etc). Sin embargo, los conceptos orientados a objetos (por ejemplo, herencia) no están suficientemente preocupados por la extensión. Se utiliza una notación de clase en el diagrama de clase para representar una página HTML. WAE se centra principalmente en la tecnología de la página de secuencias de comandos, como ASP y JSP.

Mientras que Osorio [23] y Mondragón [24] refieren que la WAE presenta una serie de estereotipos que constituyen a los elementos WEB, los mismos que pueden ser formularios, enlaces, páginas Web entre otros. Cabe destacar que a pesar de la WAE contribuyó con el modelamiento de las aplicaciones Web tradicionales, aún requiere estereotipos y relaciones donde se refleje la interactividad, cookies, comunidades móviles, redes sociales y otras notaciones que se aplican hoy en día para las aplicaciones Web.

\subsection{FASES DE LA METODOLOGÍA WAE}

Según el análisis de Osorio [23], la metodología WAE cuenta son las siguientes fases como se indica en la ilustración.

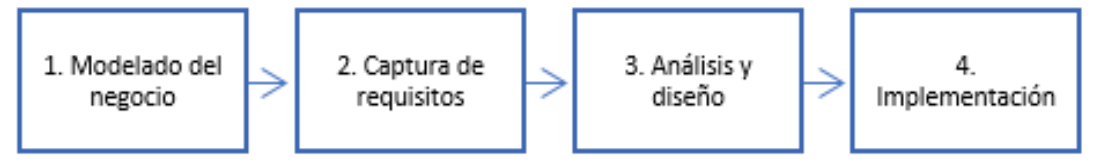

Ilustración 4. Fases de la metodología WAE.

- Modelado del negocio: comprende el flujo de actividades que se realizan dentro de la organización, en otras palabras, aquí se describen cuáles son los departamentos, empleados y la interacción que existe entre ellos. 
- Captura de requisitos: búsqueda de los requerimientos válidos y necesarios para el desarrollado de la aplicación, al mismo tiempo que los simboliza de forma adecuada para los desarrolladores, usuarios y clientes.

- Análisis y diseño: análisis de todos los requerimientos que se obtuvieron en la fase anterior con lograr un entendimiento mucho más claro de lo que se pretende con el sistema. Como productos de esta esta fase se crea diagramas de secuencia, componentes y clases.

- Implementación: fase final en la que se abarca la arquitectura física sobre la cual se implementará la aplicación y su entorno.

\subsection{IWEB (INGENIERÍA WEB)}

Según Texier \& Bermúdez [25] IWeb demanda un proceso de software incremental y evolutivo. Pressman también señala que el modelo en las primeras versiones puede ser un modelo en papel o un prototipo, y durante las últimas iteraciones se producen versiones cada vez más completas del sistema diseñado.

"La IWeb se divide en un número de actividades estructurales, también llamadas regiones de tareas. Generalmente, existen entre tres y seis regiones de tareas, las cuales no necesariamente se deben aplicar todas por cada iteración". [25]. IWeb es una metodología que se enfoca en la creación de aplicación y sistemas Web de alta calidad, basándose en principios científicos de ingeniería. Dichas aplicaciones hacen posible el acceso desde ordenadores remotos.

\subsection{FASES DE LA METODOLOGÍA IWEB}

A continuación, citaremos las siete fases de la metodología IWeb.

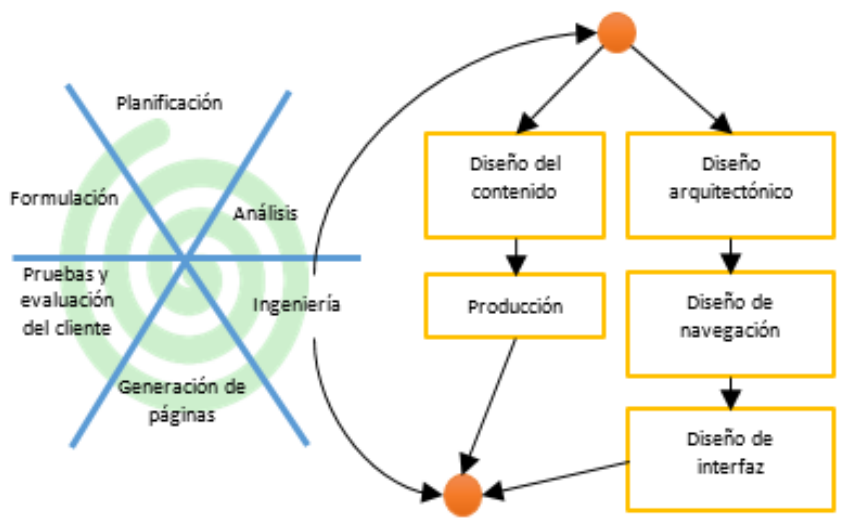

Ilustración 5. Fases de la metodología IWEB.

- Formulación: En esta primera fase se identifican los objetivos, metas, se establece el alcance de la aplicación y su primera entrega. Aspectos muy importantes a considerar en esta fase es conocer la motivación para el desarrollo de la aplicación, verificar si es necesaria o no, e identificar quien la va a utilizar. 
- Planificación: estimar el coste general del proyecto a realizar, así como también planes de contingencia debido a posibles riesgos, el ámbito y describir la calidad y gestión de la aplicación en cuanto a cambios.

- Análisis: establecer los requerimientos de diseño y técnicos, también se analiza el contenido del mismo, su iteración, funcionalidad y configuración.

- Ingeniería: lograr la integración del diseño arquitectónico, de navegación y de interfaz. Es por esto que se divide en diseño del contenido, producción, diseño arquitectónico diseño de navegación, y diseño de interfaz

- Generación de páginas: se integran los diseños de la etapa anterior a través de herramientas como lenguajes de programación y etiquetado que sirvan como base la construcción de la aplicación Web.

- Pruebas o test: se comprueba la lógica de negocios aplicada en el sistema, y se verifican las entradas y salidas de datos con el fin de descubrir errores de funcionalidad, comportamiento o rendimiento.

- Evaluación de cliente: permite corregir errores gracias a las iteraciones realizadas con el fin de ir puliendo la aplicación en comparación a las iteraciones anteriores.

\section{METODOLOGÍA}

La investigación a realizarse es de tipo bibliográfica, analítica y de campo, la misma que permitirá obtener información relevante respecto a las metodologías para el desarrollo de aplicaciones Web. Para realizar el siguiente trabajo se han denominado diferentes etapas que se centrarán en la comparación de las metodologías en aplicaciones Web, las cuales son:

1. Investigación bibliográfica de trabajos que correspondan con estudios similares desarrollados en el mismo campo (metodologías para el desarrollo de aplicaciones Web), además empresas y programadores de la provincia del Oro que se encuentren dentro de este ámbito.

2. Determinación y procesamiento de datos recopilados.

3. Establecimiento de un análisis comparativo de las diferentes metodologías en el desarrollo en aplicaciones Web.

4. Análisis de los datos obtenidos y verificación de los mismos.

5. Elaboración de los resultados y la conclusión sobre metodologías en aplicaciones Web, selección de la mejor alternativa.

\section{RESULTADOS}

A continuación, se muestra una serie de comparativas de las metodologías cada una de ellas con diferentes criterios que abarcan aspectos de requisitos, desarrollo, diseño, y calidad. En la tabla 2, se muestra una comparación de los requisitos que contempla cada metodología estudiada. 
DOI: http://dx.doi.org/10.17993/3ctecno.2018.v7n1e25.1-19

\begin{tabular}{|c|c|c|c|c|c|c|}
\hline \multirow{2}{*}{ Requerimientos } & \multicolumn{7}{|c|}{ Metodologías } \\
\cline { 2 - 7 } & WSDM & SOHDM & OODHM & UWE & WAE & IWEB \\
\hline Datos & $\mathrm{X}$ & $\mathrm{x}$ & $\mathrm{x}$ & $\mathrm{x}$ & $\mathrm{x}$ & $\mathrm{x}$ \\
\hline Interfaz de usuario & & $\mathrm{x}$ & $\mathrm{x}$ & $\mathrm{x}$ & $\mathrm{x}$ & $\mathrm{x}$ \\
\hline Navegacionales & & & $\mathrm{x}$ & $\mathrm{x}$ & $\mathrm{x}$ & $\mathrm{x}$ \\
\hline Personalización & $\mathrm{x}$ & & $\mathrm{x}$ & & & \\
\hline Transaccionales & & $\mathrm{x}$ & & $\mathrm{x}$ & & \\
\hline No funcionales & $\mathrm{x}$ & $\mathrm{x}$ & $\mathrm{x}$ & $\mathrm{x}$ & $\mathrm{x}$ & $\mathrm{x}$ \\
\hline
\end{tabular}

Tabla 2. Comparación de requisitos en el entorno Web contemplados en las metodologías.

Analizando los resultados y teniendo en cuenta que las metodologías, se encuentran ordenadas en orden cronológico. Se puede evidenciar que en un principio las metodologías solo se centraban en los datos y la interfaz que se le proporcionaba al usuario mientras que en las más actuales se resalta la importancia de tratar los requisitos de personalización, navegación, transaccionales y no funcionales. Así mismo es importante mencionar que la metodología OOHDM y la UWE son las que contempla la mayoría de los requisitos en el entorno Web.

La tabla 4 muestra un análisis comparativo entre las diferentes metodologías, sus técnicas, la notación y sus herramientas de soporte. Se estableció abreviaturas en la tabla 3, con el fin de hacer más entendible los nombres de las metodologías [26].

\begin{tabular}{|c|l|}
\hline $\mathbf{N} N$ & Abreviaturas o Acrónimos \\
\hline $\mathbf{1}$ & Entity-Relationship, Entidad - Relación (E-R) \\
\hline $\mathbf{2}$ & Object Oriented, Orientado a Objetos (OO) \\
\hline $\mathbf{3}$ & Relationship Management Data Model, Modelo de Datos de Administración de Relaciones (RM DM) \\
\hline $\mathbf{4}$ & Graphical User Interface, Interfaz de Usuario Gráfica (GUI) \\
\hline $\mathbf{5}$ & Abstract Data View, Vista de Datos Abstracta (ADV) \\
\hline $\mathbf{6}$ & Object Modeling Technique, Técnica de Modelado de Objetos (OMT) \\
\hline $\mathbf{7}$ & Unified Modeling Language, Lenguaje de Modelado Unificado (UML) \\
\hline
\end{tabular}

Tabla 3. Abreviaturas de la tabla 4. 
Área de Innovación y Desarrollo, S.L.

ISSN: $2254-4143$

DOI: http://dx.doi.org/10.17993/3ctecno.2018.v7n1e25.1-19

\begin{tabular}{|c|c|c|c|c|}
\hline Metodología & $\begin{array}{l}\text { Técnica de } \\
\text { modelado }\end{array}$ & Representación gráfica & Notación & Herramienta de soporte \\
\hline OOHDM & 00 & $\begin{array}{l}\text { 1. Diagrama de clases } \\
\text { 2. Diagrama navegacional, clase + contexto } \\
\text { 3. Diagrama de configuración de ADV y Diagrama ADV }\end{array}$ & $\begin{array}{l}\text { 1. OMT/UML } \\
\text { 2. ADVs }\end{array}$ & OOHDM-Web \\
\hline SOHDM & $\begin{array}{l}\text { Escenarios } \\
\text { Vistas-OO }\end{array}$ & $\begin{array}{l}\text { 1. Diagramas de escenarios de actividad } \\
\text { 2. Diagrama de estructura de clase } \\
\text { 3. Vista } 00 \\
\text { 4. Esquema de enlace navegacional } \\
\text { 5. Esquema de páginas }\end{array}$ & Propio & \\
\hline WSDM & $E-R / O O$ & $\begin{array}{l}\text { 1. Diagrama de } E-R \text { o clase } \\
\text { 2. Capas de navegación }\end{array}$ & $\begin{array}{l}\text { 1. } \mathrm{E}-\mathrm{R} / \mathrm{OMT} \\
\text { 2. Propio }\end{array}$ & \\
\hline WAE & $\mathrm{OO}$ & Diagramas UML & UML & Rational Rose \\
\hline UWE & 00 & Diagramas UML & UML & Magic Draw (Magic UWE \\
\hline IWEB & $\mathrm{E}-\mathrm{R} / \mathrm{OO}$ & $\begin{array}{l}\text { 1. Esquema navegacional } \\
\text { 2. Esquema arquitectónico. }\end{array}$ & UML & XML, HTML \\
\hline
\end{tabular}

Tabla 4. Comparación de las metodologías en el desarrollo de aplicaciones Web [26].

La tabla 5 muestra una comparación de diseño basados en los tres niveles típicos del desarrollo Web: conceptual, estructural y visible.

\begin{tabular}{|c|c|c|c|}
\hline Metodología & Nivel concepto & Nivel estructura & Nivel visible \\
\hline OOHDM & $\begin{array}{l}\text { Clases } \\
\text { Perspectiva } \\
\text { Relación-0O }\end{array}$ & $\begin{array}{l}\text { Enlace } \\
\text { Clase navegacional } \\
\text { Contexto navegacional }\end{array}$ & $\begin{array}{l}\text { ADV } \\
\text { En contexto. }\end{array}$ \\
\hline SOHDM & $\begin{array}{l}\text { Escenarios: } \\
\text {-Evento } \\
\text {-Actividad } \\
\text { Flujo de actividad }\end{array}$ & $\begin{array}{l}\text { Enlace navegacional } \\
\text { Visita-0O: } \\
\text { - Base } \\
\text { - Asociación }\end{array}$ & $\begin{array}{l}\text { Componente Ul: } \\
\text { - Elección } \\
\text { - Texto de entrada de búsqueda } \\
\text { - Botón }\end{array}$ \\
\hline WSDM & $\begin{array}{l}\text { Objeto } \\
\text { Perspectiva } \\
\text { Relación }\end{array}$ & $\begin{array}{l}\text { Enlace } \\
\text { Componente } \\
\text { - Navegación } \\
\text { - Información } \\
\text { - Externo } \\
\text { Camino navegacional }\end{array}$ & No contiene. \\
\hline WAE & $\begin{array}{l}\text { CASE } \\
\text { Relación-0O }\end{array}$ & $\begin{array}{l}\text { Enlace } \\
\text { Enlace dirigido } \\
\text { Redirigir } \\
\text { Construir } \\
\text { Enviar }\end{array}$ & $\begin{array}{l}\text { Conjunto de marcos } \\
\text { Formulario }\end{array}$ \\
\hline UWE & $\begin{array}{l}\text { Meta-modelo } \\
\text { OO } \\
\text { Escenarios Web }\end{array}$ & $\begin{array}{l}\text { Modelo lógico } \\
\text { y Navegacional } \\
\text {-Asociación } \\
\text {-Interacción temporal } \\
\end{array}$ & Vistas representas por diagramas UML. \\
\hline IWEB & Proceso ágil & -Navegación & WebApps \\
\hline
\end{tabular}

Tabla 5. Comparación de conceptos de diseño de las metodologías de desarrollo Web [26].

En base a la comparación realizada previamente y con fundamentos de la investigación realizada por Silva [26], se puede decir que la metodología más utilizada en el desarrollo de aplicaciones Web es SOHDM, tomando en cuenta que este método ofrece procesos más seguros y enfocados a aspectos de métricas de calidad que verifican que las aplicaciones tengan mayor confiabilidad, consistencia y seguridad. 
En la tabla 6 se muestra una comparación del criterio de calidad basado en la norma ISO/IEC 9126 con las distintas metodologías que ha estudiado.

\begin{tabular}{|c|c|c|c|c|c|c|}
\hline \multirow{2}{*}{ Criterios } & \multicolumn{6}{|c|}{ Metodologías } \\
\hline & Оорнм & SOHDM & WSDM & WAE & UWE & IWEB \\
\hline Funcionalidad & $\begin{array}{c}\text { Adecuación } \\
\text { Interoperabilidad }\end{array}$ & $\begin{array}{c}\text { Adecuación } \\
\text { Interoperabilidad }\end{array}$ & $\begin{array}{c}\text { Adecuación } \\
\text { Interoperabilidad }\end{array}$ & $\begin{array}{c}\text { Adecuación } \\
\text { Interoperabilidad } \\
\text { Seguridad }\end{array}$ & $\begin{array}{c}\text { Adecuación } \\
\text { Interoperabilidad } \\
\text { Seguridad }\end{array}$ & $\begin{array}{c}\text { Adecuación } \\
\text { Interoperabilidad } \\
\text { Seguridad }\end{array}$ \\
\hline Fiabilidad & Recuperabilidad & Recuperabilidad & Baja & Baja & Recuperabilidad & Baja \\
\hline Usabilidad & $\begin{array}{l}\text { Aprendizaje } \\
\text { Comprensión } \\
\text { Operatividad }\end{array}$ & Operatividad & Operatividad & Baja & $\begin{array}{l}\text { Aprendizaje } \\
\text { Comprensión } \\
\text { Operatividad }\end{array}$ & Baja \\
\hline Eficiencia & Media & Media & Media & Baja & Baja & Baja \\
\hline Portabilidad & Adaptabilidad & Adaptabilidad & Adaptabilidad & $\begin{array}{l}\text { Adaptabilidad } \\
\text { Coexistencia }\end{array}$ & Adaptabilidad & $\begin{array}{l}\text { Adaptabilidad } \\
\text { Coexistencia }\end{array}$ \\
\hline Mantenibilidad & $\begin{array}{c}\text { Facilidad de } \\
\text { análisis, cambio } \\
\text { y pruebas }\end{array}$ & Media & Media & Baja & $\begin{array}{c}\text { Facilidad de } \\
\text { análisis y cambio }\end{array}$ & Media \\
\hline
\end{tabular}

Tabla 6. Comparación de criterios de calidad de las metodologías de desarrollo Web.

Como se puede observar en la tabla, la mayoría de las características según los criterios de calidad establecidos por la ISO/IEC 9126 [27] son abarcados por la metodología OOHDM, ya que presenta una mejor adaptabilidad frente a cambios, es de fácil aprendizaje, y permite la rápida corrección de errores y problemas presentados a lo largo del ciclo de vida.

Es importante mencionar que también se realizó un estudio de campo el cual tuvo como área piloto de estudio a los programadores y empresas desarrolladoras de software de la provincia de El Oro según la INEC (Instituto Nacional de Estadísticas y Censos) [28]. En la ilustración 6 podemos observar que del total área de estudio, el $74 \%$ manifiesta que realizan aplicaciones Web, en relación a un $26 \%$ que se encargan también del desarrollo de software, pero no en esa área. 


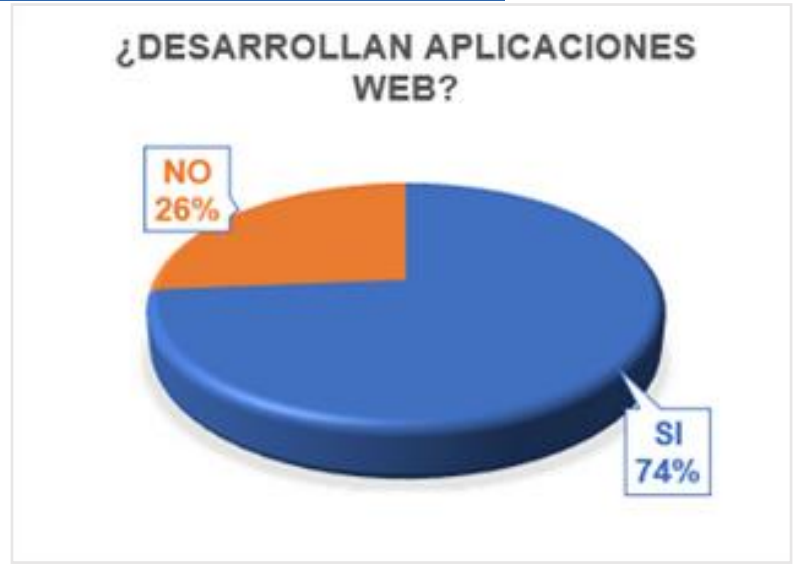

llustración 1. Pregunta 1.

En otra de las interrogantes que fueron presentadas se determinó el porcentaje de empresas y programadores localizados en la provincia que desarrollan aplicaciones Web y que además utilizan o implementan alguna metodología que soporte el proceso o guíe el ciclo de vida del software, ante lo cual un $57 \%$ manifestó que, si aplican metodologías, mientras que un $43 \%$ no aplica tal como se puede observar en la ilustración 7.

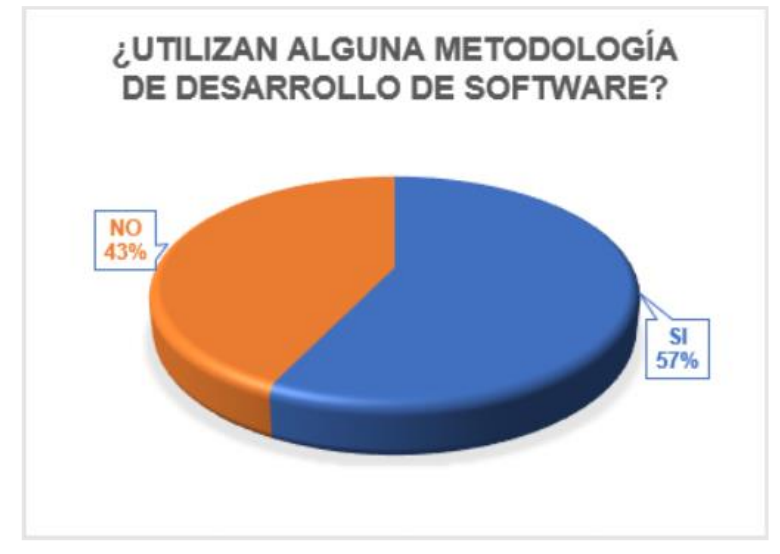

Ilustración 2. Pregunta 2.

En la ilustración 8 se puede evidenciar que la metodología que se aplica de forma mayoritaria en la OODHM, debido a la fácil adaptabilidad para cualquier tipo de proyecto, respuesta inmediata ante problemas o errores existen dentro del ciclo de vida, así como a su fácil implementación y operatividad dentro de cada proyecto. Otras de las metodologías que también se implementa es UWE debido al proceso de diseño iterativo e incremental que permite adaptar nuevos requerimientos y peticiones dentro del proyecto. 


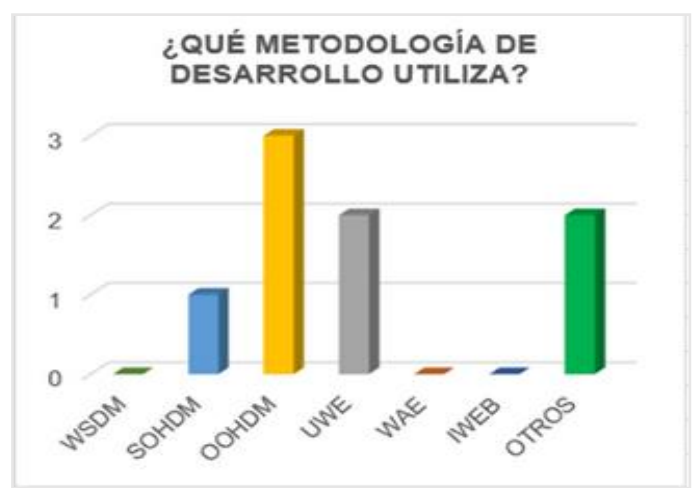

Ilustración 3. Pregunta 3.

\section{DISCUSIÓN}

Las metodologías para el desarrollo de software Web cumplen un papel importante en cuanto al desarrollo e implementación de este tipo de aplicaciones, además, existen distintas metodologías de desarrollo de software Web que pueden ser aplicados a la construcción de software. No hay una metodología mejor que otra, sino que cada una se adapta a unas características particular del producto a obtener, las más conocidas según la investigación realizada son: SOHDM, OOHDM, WSDM, WAE, UWE y IWEB. [29] [30]

La metodología OOHDM es la que más se utiliza para el proceso de desarrollo de aplicaciones Web debido a su fácil adaptabilidad en todo proyecto. Es importante también mencionar que existe un margen muy pequeño de diferencia entre las empresas y desarrolladores que aplicación metodología como las que no aplican, razón por la cual es estudios posteriores se pretende hacer énfasis en el uso de las metodologías como marco de trabajo indispensable para la creación de productos de software de todo tipo.

\section{CONCLUSIONES}

A través de la información presentada se concluye que:

En primordial que los desarrolladores realicen software por medio de metodologías que permitan guiar su trabajo y acoplar el producto final con aspectos como la funcionalidad, seguridad, consistencia y fiabilidad, los cuales permiten que el sistema esté acorde a normas de calidad y cumpla con el objetivo por el cual fue creado.

Independientemente de la metodología que el profesional escoja se debe efectuar las diversas tareas expuestas en los apartados anteriores que aseguraran un producto eficiente y cuyas funcionalidades estén acorde a lo establecido previamente por el cliente.

La comparativa efectuada permitió determinar que le metodología OOHDM ofrece un marco de trabajo óptimo para el desarrollo de aplicaciones Web, ya que facilita el trabajo dentro del equipo desarrollador y agiliza los procesos optimizando sus etapas, además de contemplar más etapas en el ciclo de vida de desarrollo y precisa el modelado de objetos. 


\section{REFERENCIAS BIBLIOGRÁFICAS}

[1] C. d. P. Heredero, Informática y comunicaciones en la empresa, illustrated, Ed., Madrid: ESIC, 2004.

[2] R. Noriega Martínez, El Proceso de Desarrollo de Software: 2a Edición, Segunda ed., Smashwords Edition , 2017.

[3] D. Ramos Cardozzo, Desarrollo de Software: Requisitos, Estimaciones y Análisis., Segunda ed., Createspace Independent Pub, 2016.

[4] R. S. Pressman, Ingeniería del Software. Un enfoque práctico, Séptima ed., México: Mc Graw-Hill, 2010.

[5] H. E. Traverso, L. B. Prato, L. N. Villoria, G. A. Gomez Rodriguez, C. Priegue, R. M. Caivano y M. L. Fissore, "Herramientas de la Web 2.0 aplicadas a la educación.," VIII Congreso de Tecnología en Educación y Educación en Tecnología, no 8, pp. 1-8, 2013.

[6] L. Mora, Programación de aplicaciones Web: historia, principios básicos y clientes Web, Primera ed., San Vicente: Club Universitario, 2002.

[7] A. Ramos Martín y M. J. Ramos Martín, Aplicaciones Web, Segunda ed., Madrid: Ediciones Paraninfo, S.A., 2014.

[8] L. Fernández y P. Bernad, "Gestión de riesgos en proyectos de desarollo de software en España: estudio de la situación,» Facultad de Ingeniería Universidad de Antioquia, vol. I, no 70, pp. 223-243, 2014.

[9] E. Delgado, «Metodologías de desarrollo de software. ¿Cuál es el camino?,» Revista de Arquitectura e Ingeniería, vol. II, no 3, pp. 1-7, 2008.

[10] I. Sommerville, Ingeniería del Software, Séptima ed., Madrid: Pearson Educación, 2005.

[11] I. Leiva y M. Villalobos, «Método ágil híbrido para desarrollar sofware en dispositivos móviles,» Chilena de ingeniería, vol. XIII, no 3, pp. 473-488, 2015.

[12] A. Navarro, J. Fernández y J. Morales, «Revisión de metodologías ágiles para el desarrollo de software,» Prospectiva, vol. XI, no 2, pp. 30-39, 2013.

[13] P. Letelier, C. Penadés y J. Canós, «Métodologías ágiles para el desarrollo de software: eXtreme Programming (XP),» Ciencia y Técnica Administrativa, vol. VI, no 26, pp. 1-6, 2006.

[14] S. D. Orantes Jiménez, «Metodologías híbridas para desarrollo de software: una opción factible para México,» Revista Digital Universitaria, vol. XIII, no 1, pp. 3-17, 2017.

[15] A. Nieves del Valle, «Metodologías de diseño usadas en ingeniería Web, su vinculación con las NTICs, " Universidad Nacional de la Plata, Madrid, 2010.

[16] A. Mishra, "Benefits of combining WSDM and Scrum Framework development," International Journal of Scientific \& Technology research, vol. III, pp. 328-330, 2014. 
[17] T. Appelmans, «Web Globalization and WSDM Methodology of Web Design,» Vrije Universiteit Brussel, Bruselas, 2004.

[18] Y. J. Marcano Aular y R. Talavera Pereira, «Metodologías para el desarrollo de ambientes de aprendizaje en entornos colaborativos: Una reflexión teórica, » Redalyc, vol. VII, no 1, pp. 63-71, 2007.

[19] D. F. Flores Chicaíza y F. D. Jiménez Paredes, «Sistema de Gestión de E-Portafolios de Aprendizaje para la carrera de Ingeniería de Sistemas e Informática Utilizando UWEUML.,» Universidad de las Fuerzas Armadas, Sangolquí, 2015.

[20] «La utilización de la Ingeniería de Software en hipermedia,» Ciencia UNEMI, vol. VI, pp. 102-117, 20171.

[21] M. J. Escalona, Ingeniería de Requisitos en Aplicaciones para la Web - Un estudio comparativo, Sevilla: Universidad de Sevilla, 2002.

[22] R. SotoDe Giorgis, W. Palma Muñoz y S. Roncagliolo De La Horra, «Propuesta de un modelo navegacional para el desarrollo de aplicaciones basadas en OOHDM, » vol. I, no 1, pp. 1-10, 2011.

[23] M. J. Osorio Bastidas, «Desarrollo de una solución E-Comerce B2E para la gestión de pedido a proveedores en la empresa Segundo Eloy Corrales e Hijos SECOHI Cia Itada.," Pontifica Universidad Católica del Ecuador, Ambato, 2009.

[24] A. Mondragon Valencia, "Modelo de Navegación Web para dispositivos móviles"WAE FOR MOBILE DEVICES",» Universidad del Valle, Santiago de Cali, 2014.

[25] J. D. Texier y M. Bermúdez, "Un Sistema de Escritura de Traductores de Escritura Vía Web," International Latin American and Caribbean Conference for Engineering and Technology, vol. I, no 1, pp. 1-9, 20008.

[26] D. A. Silva y B. Mercerat, "Construyendo aplicaciones Web con una metdología de diseño orientada a objetos," Revista Colombiana de Computación, vol. II, no 2, p. 20, 2001.

[27] R. L. Granados La Paz, Despliegue y puesta en funcionamiento de componentes software. IFCT0609, Primera ed., IC Editorial, 2015.

[28] «Instituto Nacional de Estadístcas y Censos,» Gobierno Nacional de la República del Ecuador, 2016. [En línea]. Available: www.ecuadorencifras.gob.ec/. [Último acceso: 01 Enero 2018].

[29] C. G. Nieves-Guerrero, J. P. Ucán-Pech y V. H. Menéndez-Domínguez, "UWE en Sistema de Recomendación de Objetos de Aprendizaje. Aplicando Ingeniería Web: Un Método en Caso de Estudio," Revista Latinoamericana de Ingeniería de Software, vol. I, no 1, pp. 137-143, 2014.

[30] A. Narváez, P. Baldeón, C. Hinojosa y D. Martínez, «Experiencia de desarrollo de una aplicación Web utilizando la metodología UWE y lenguaje QVT en la transformación de modelos., " vol. I, no 1, pp. 1-10, 2012. 